\title{
miR-222 regulates brain injury and inflammation following intracerebral hemorrhage by targeting ITGB8
}

\author{
YAN-YAN BAI ${ }^{1}$ and JUN-ZHI NIU ${ }^{2}$ \\ ${ }^{1}$ Department of Neurology, The First Hospital of Yulin, Yulin, Shaanxi 719000; \\ ${ }^{2}$ Department of Information, Jining No. 1 People's Hospital, Jining, Shandong 272011, P.R. China
}

Received December 22, 2018; Accepted July 30, 2019

DOI: $10.3892 / \mathrm{mmr} .2019 .10903$

\begin{abstract}
Intracerebral hemorrhage (ICH) is a disease associated with high mortality and morbidity. MicroRNAs (miRNAs) have been reported to be associated with the pathogenesis of numerous cerebrovascular diseases, including ICH. miR-222 has been revealed to play important roles in various physiological and pathological processes in cardiovascular diseases. However, its role in ICH remains largely unknown. The aim of the present study was to evaluate the potential effect of miR-222 on brain injury in ICH. The results revealed that the expression of miR-222 was significantly increased in $\mathrm{ICH}$, and downregulation of miR-222 significantly reduced erythrocyte lysate-induced cell apoptosis by decreasing the levels of cleaved caspase-3, cleaved caspase- 9 and Bax and increasing the level of Bcl-2. In addition, downregulation of miR-222 suppressed the inflammatory responses in erythrocyte lysate-induced microglia, and inhibited inflammation, brain water content and improved neurological functions in ICH mice. Mechanistically, integrin subunit $\beta 8$ (ITGB8) was identified as a direct target of negative regulation by miR-222 in microglia cells, and up-regulation of ITGB8 led to the attenuation of inflammation and apoptosis. Collectively, the present findings indicated that miR-222 was a crucial regulator of inflammation via targeting of ITGB8, and represented a promising therapeutic strategy for $\mathrm{ICH}$.
\end{abstract}

\section{Introduction}

Intracerebral hemorrhage ( $\mathrm{ICH})$ is the most common human cerebrovascular disease, accounting for $10-15 \%$ of strokes (1), which is characterized by rupture of one or more blood vessels in the brain and infiltration of blood into the brain (2). Hematoma after ICH disrupts neighboring blood vessels,

Correspondence to: Dr Jun-Zhi Niu, Department of Information, Jining No. 1 People's Hospital, 6 Health Road, Jining, Shandong 272011, P.R. China

E-mail: niujunzhi1@163.com

Key words: microRNA-222, intracerebral hemorrhage, inflammatory, apoptosis, integrin subunit $\beta 8$ increases bleeding and hematoma growth, which cause ischemia and impaired neuronal function (3). Recent studies have demonstrated that ICH triggers an inflammation cascade to accelerate the formation of edema, which aggravates the mass effect around hematoma and amplifies cell death $(4,5)$. Inflammatory cells, such as blood-derived leukocytes, microglia and macrophages, are essential for removing the hematoma but also increase brain damage caused by cerebral hemorrhage (6). Microglia are thought to be the earliest inflammatory cells of $\mathrm{ICH}$ (7), and are the major cell type responsible for damage after ICH by releasing cytokines, chemokines, ferrous iron and other immunoactive molecules (8).

MicroRNAs (miRNA) are endogenous small RNA molecules, which contribute to post-transcriptional gene silencing and regulate the expression of hundreds of target genes (9). They can control diverse fundamental biological and pathological processes, such as growth, development and death (10). Recent studies found that miRNAs could regulate the transcription of microglia and the expression levels of related signaling pathways, and participate in the polarization of microglia $(11,12)$. For example, it was revealed that overexpression of miRNA-126-3p could attenuate blood-brain barrier disruption, cerebral edema and neuronal injury following $\mathrm{ICH}$ by regulating PIK3R2 and Akt (13). In addition, miR-124 could ameliorate ICH-induced inflammatory injury by modulating microglia polarization toward the $\mathrm{M} 2$ phenotype via C/EBP- $\alpha$ (14).

miR-222 is a component of the miR221/222 cluster, whose members are located on the X chromosome (XP113) with the same seed sequence (15). miR-222 is associated with the pathogenesis and progression of cancer and functions by affecting cell proliferation, tumor growth and cell apoptosis $(16,17)$. However, the exact mechanism of miR-222 on inflammation and brain injury in ICH has not been elucidated. In the present study, the miR-222 level was detected, and the potential role of miR-222 in $\mathrm{ICH}$ in vivo and in vitro was further explored. It is expected that miR-222 may provide new insights into the understanding of cerebral protection and act as a neuroprotective agent for ICH therapeutics.

\section{Materials and methods}

Primary cell cultures. For primary microglia cells, glial cells were isolated from the brains of rat pups and cultured in DMEM (Gibco; Thermo Fisher Scientific, Inc.) supplemented 
with $20 \%$ fetal bovine serum (Invitrogen; Thermo Fisher Scientific, Inc.) in a humidified atmosphere at $37^{\circ} \mathrm{C}$. Microglia was isolated from the mixed glial population when mixed glial cells were confluent (12-14 days). The purity of microglia was assessed by immunofluorescence using microglia-specific antibody CDb11. Microglial cultures with $>98 \%$ purity were used for the study.

Animals. In total, 60 male C57BL/6 mice (6-8 weeks, 18-22 g) were purchased from Model Animal Research Institute of Nanjing University and bred under specific pathogen-free conditions. Experiments were performed according to animal care guidelines approved by The Animal Ethics Committee of Nanjing Medical University, and animals were treated in accordance with The Guidelines of the United States National Institutes of Health. Mice were maintained at constant ambient temperature $\left(22 \pm 1^{\circ} \mathrm{C}\right)$ under a 12 -h light/dark cycle with food and water ad libitum. The model of mice was established as described (18). Animal health and behavior were monitored every day.

Preparation of erythrocyte lysates. Blood was obtained from C57BL/6 mice. Single-cell suspension of erythrocytes was prepared. Then, $2 \times 10^{5}$ erythrocytes were incubated with $1 \mathrm{ml}$ red blood cell lysing solution for $20 \mathrm{~min}$, and centrifuged at $2,000 \mathrm{x} \mathrm{g}$ and $4^{\circ} \mathrm{C}$ for $10 \mathrm{~min}$. Subsequently, the supernatants were used as erythrocyte lysates.

Cell transfection. The miR-222 mimics, inhibitors, and corresponding negative control (NC) were all purchased from Shanghai GenePharma Co., Ltd. The sequence of the miR-222 mimics was 5'-AGCUACAUCUGGCUACUG GGU-3', the corresponding NC was 5'-UUCUCCGAACGU GUGUCACGUTT-3', the miR-222 inhibitor was 5'-ACC CAGUAGCCAGAUGUAGCU-3' and the corresponding NC was 5'-UCUACUCUUUCUAGGAGGUUGUGA-3'. Cells were seeded into 6-well plates and cultured for $24 \mathrm{~h}$, and then transfected with miR-222 mimics, miR-222 inhibitors or corresponding NC $(20 \mu \mathrm{M})$ using a Lipofectamine ${ }^{\circledR} 3000$ Transfection kit (Invitrogen; Thermo Fisher Scientific, Inc.). After $48 \mathrm{~h}$, the cells were used for further assays.

Reverse transcription-quantitative PCR (RT-qPCR). For mRNA quantification, total RNA was isolated from cells with TRIzol ${ }^{\circledR}$ (Invitrogen; Thermo Fisher Scientific, Inc.) according to the manufacturer's protocols. Amplification and quantification of cDNA were carried out with SYBR Green ROX Mix (ABgene). RT-qPCR was performed using SYBR Premix Ex Taq ${ }^{\mathrm{TM}}$ (Takara Bio, Inc.) on a LightCycler 480 system (Roche Diagnostics), using the following cycling conditions: $95^{\circ} \mathrm{C}$ for $10 \mathrm{~min}, 95^{\circ} \mathrm{C}$ for $10 \mathrm{sec}, 60^{\circ} \mathrm{C}$ for $20 \mathrm{sec}$, $72^{\circ} \mathrm{C}$ for $10 \mathrm{sec}, 40$-sec cycles. GAPDH and U6 were used as internal controls to normalize the expression levels of mRNA and miRNAs (19), respectively. The sequences for the primers used were as follows: miR-222, 5'-AGCTACATCTGGCTA CTGGGT-3'; U6, 5'-CAAGGATGACACGCAAATTCG-3'; GAPDH forward, 5'-GGTCACCAGGGCTGCTTTTA-3' and reverse, 5'-GAGGGATCTCGCTCCTGGA-3'. The primers used were purchased from Generay Biotech. The relative expression was calculated by the $2^{-\Delta \Delta \mathrm{Cq}}(20)$.
Cell Counting Kit-8(CCK-8) assay. Cell viability of microglia was assessed using a CCK-8 assay. Cells $\left(1 \times 10^{4}\right)$ were seeded in 96-well plates. After being cultured for $24 \mathrm{~h}, 10 \mu \mathrm{l}$ erythrocyte lysates or PBS was added the cell culture medium. After $48 \mathrm{~h}$, the culture supernatant was removed and the cells were incubated with $10 \mu \mathrm{l} \mathrm{CCK-8} \mathrm{(Dojindo} \mathrm{Molecular} \mathrm{Technologies,}$ Inc.) reagent for $4 \mathrm{~h}$ at $37^{\circ} \mathrm{C}$, according to the manufacturer's protocol. Then, the absorbance was detected at a wavelength of $490 \mathrm{~nm}$ on a microplate reader (Multiskan MK3; Thermo Fisher Scientific, Inc.).

Cell apoptosis. Apoptosis was evaluated using an apoptosis and necrosis assay kit (Oncogene Research Products). Briefly, cells were suspended in binding buffer and incubated at room temperature in the dark for $15 \mathrm{~min}$. Annexin-V-fluorescein isothiocyanate and propidium iodide were added to cell suspension for $15 \mathrm{~min}$ in the dark at room temperature according to the manufacturer's protocol. Then, stained cells were analyzed using a FC500 flow cytometer equipped with Cell Quest 3.0 software (BD Biosciences).

Western blotting. Briefly, total protein was extracted using RIPA lysis buffer (Beyotime Institute of Biotechnology) from cells or tissues and quantified using a commercial bicinchoninic acid kit (BCA protein Assay kit; Pierce; Thermo Fisher Scientific, Inc.). Protein $(30 \mu \mathrm{g})$ was separated by SDS-PAGE on $12 \%$ gels and transferred onto PVDF membranes. Then, the membrane was blocked with $5 \%$ non-fat milk and incubated with primary antibodies overnight at $4^{\circ} \mathrm{C}$, including cleaved caspase-3 (cat. no. 9661; 1:1,000), cleaved caspase-9 (cat. no. 9505; 1:1,000), Bcl-2 (cat. no. 2872; 1:1,000), Bax (cat. no. 2774; 1:1,000) and GAPDH (cat. no. 8884; 1:2,000; all from Cell Signaling Technology, Inc.). The blots were incubated for $2 \mathrm{~h}$ at room temperature with horseradish peroxidase-conjugated secondary antibodies (cat. no. 7076; 1:2,000; Cell Signaling Technology, Inc.). The signals were detected with an ECL system (Beyotime Institute of Biotechnology) and analyzed using the Quantity One software 4.6.6 (Bio-Rad Laboratories, Inc.).

ELISA. The levels of inflammatory factors in cell supernatants were measured by ELISA according to the manufacturer. Microglia were plated in 24-well plates and stimulated with erythrocyte lysates for $48 \mathrm{~h}$, and then culture supernatant was added and stored at $-80^{\circ} \mathrm{C}$ until analyzed by ELISA. The levels of IL-6 (cat. no. BMS213HS), TNF- $\alpha$ (cat. no. BMS223HS), MCP-1 (cat. no. BMS281INST) and IL-1 $\beta$ (cat. no. BMS224-2) were measured using an ELISA kit (eBioscience; Thermo Fisher Scientific, Inc.).

ICH model. Mice were anesthetized with an intraperitoneal injection of $350 \mathrm{mg} / \mathrm{kg}$ chloral hydrate and fixed on a mouse stereotaxic frame (Alcott Biotech Co., Ltd.). A 20- $\mu$ l volume of autologous non-anticoagulated blood was collected from the tail vein of the mouse and then implanted into the caudate nucleus at $2 \mu \mathrm{l} / \mathrm{min}$ at the following coordinates relative to bregma: $0.8 \mathrm{~mm}$ anterior, $2 \mathrm{~mm}$ left lateral and $3.5 \mathrm{~mm}$ deep. Then the needle was pulled out without blood reflux after 5 min duration and the wound was sutured. The body temperature remained at $37^{\circ} \mathrm{C}$ during the process, and the mice could 
A

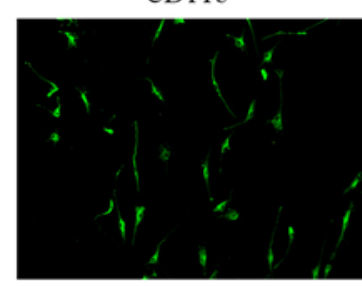

DAPI

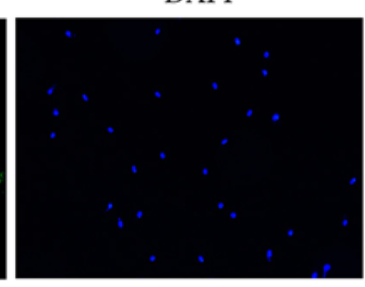

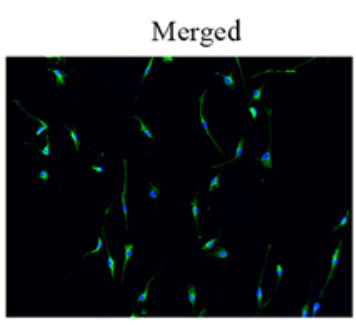

B

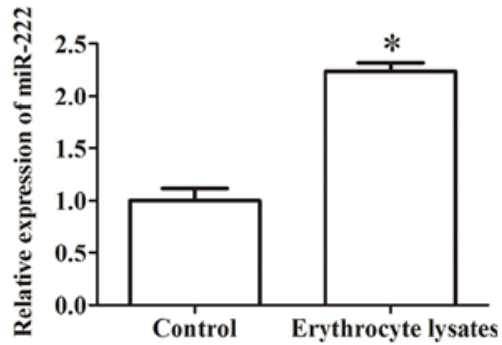

Figure 1. miR-222 is upregulated in erythrocyte lysate-induced microglia. (A) An immunofluorescence assay was used to identify microglia-specific antibody CD11b. (B) Reverse transcription-quantitative PCR assay was performed to evaluate the expression of miR-222 in erythrocyte lysate-induced microglia. Magnification, $\mathrm{x} 200$. $^{*} \mathrm{P}<0.05$ vs. the control group. miR-222, microRNA-222.

drink freely after waking up. Only the mice observed with neurological deficits were regarded as successful models.

Intracerebroventricular injection. As previously reported (14), the stereotaxic coordinates were $0.5 \mathrm{~mm}$ posterior and $1.0 \mathrm{~mm}$ lateral to bregma and 2.5-3.0 $\mathrm{mm}$ ventral to the surface of the skull. The miR-222 mimics or miR-222 inhibitor $(1.5 \mu \mathrm{l}$, $1 \mu \mathrm{g} / 1 \mu \mathrm{l})$ were added to Entranster ${ }^{\mathrm{TM}}$ in vivo transfection reagent. The solution was injected intracerebroventricularly by a micro syringe (Hamilton Company) under the guidance of the stereotaxic instrument (RWD Life Science).

Evaluation of neurological scores. The neurological deficits were determined by neurological severity scores, a composite of motor, sensory, reflex, and balance tests according to a previous study (21). Neurological function was graded on a scale of 1 to 18 ; a score of 1 point was awarded for the inability to perform the test or for the lack of a tested reflex. Scoring was conducted by 2 trained investigators, and the mean score of the subscales was the final score of each mouse.

Brain water content. The mice were decapitated and the brains were removed at $72 \mathrm{~h}$ after $\mathrm{ICH}$. The brain samples were immediately weighed on an electronic analytical balance to obtain wet weight. The brain was dried for $24 \mathrm{~h}$ at $100^{\circ} \mathrm{C}$ by an Electric Blast Drying Oven to obtain dry weight. The water content of the brain (\%) was calculated as follows $=($ Wet weight-Dry weight $) /$ Wet weight $\times 100$.

Dual luciferase 3'-UTR reporter assay. The program TargetScan (www.targetscan.org) was used to predict the target of miR-222. The wild-type and mutant integrin subunit $\beta 8$ (ITGB8) 3'-UTR dual-luciferase reporter vectors were constructed by subcloning the human ITGB 8 mRNA 3'-UTR and mutant 3'-UTR sequences into the pGL3 Dual-Luciferase Reporter Vectors (Promega Corporation). Cells were transfected with $80 \mathrm{ng}$ luciferase reporter vectors and miR-222 mimics using the Lipofectamine 3000 (Invitrogen; Thermo Fisher Scientific, Inc.). After 24 h, luciferase activities were assessed using Dual-Luciferase Reporter System (Berthold Detection Systems $\mathrm{GmbH}$ ) according to the manufacturer's instructions.

Statistical analysis. All experiments were performed at least three times. Data are presented as the mean \pm standard deviation and analyzed by GraphPad Prism 5.0 (GraphPad
Software) and SPSS 18 software (SPSS, Inc.). The differences between the two groups were calculated by Student's t-test, and the differences among multiple groups were calculated by one-way ANOVA followed by Scheffe test. $\mathrm{P}<0.05$ was considered to indicate a statistically significant difference.

\section{Results}

miR-222 is upregulated in erythrocyte lysate-induced microglia. ICH can promote microglia activation and release proinflammatory mediators, which can cause neuronal injury (22). Based on research, microglia cells were selected in response to erythrocyte lysis as a research object in vitro. For primary microglia cells, an immunofluorescence assay was conducted to identify microglia-specific antibody CD11b (Fig. 1A). Furthermore, the result of qRT-PCR revealed that the expression of miR-222 was significantly increased in the erythrocyte lysate-induced group compared with the control group, indicating that the expression of miR-222 may be related to ICH incidence (Fig. 1B).

miR-222 regulates erythrocyte lysate-induced microglia cell viability and apoptosis. To further investigate the role of miR-222 on microglia cell viability and apoptosis in vitro, miR-222 mimics or miR-222 inhibitors were transfected into microglia cells to upregulate or knockdown the level of miR-222. The result of qRT-PCR revealed miR-222 mimics significantly promoted the expression of miR-222, while miR-222 inhibitors significantly inhibited it (Fig. 2A).

Furthermore, a CCK-8 assay was conducted to evaluate microglia cell viability. As revealed in Fig. 2B, overexpression of miR-222 significantly suppressed erythrocyte lysate-induced microglia cell viability, while inhibition of miR-222 significantly promoted erythrocyte lysate-induced microglia cell viability (Fig. 2B). In addition, the effect of miR-222 on cell apoptosis was evaluated by flow cytometric analysis. The result demonstrated that the percentage of erythrocyte lysate-induced apoptotic cells was statistically increased by overexpression of miR-222, while miR-222 inhibitors significantly decreased erythrocyte lysate-induced cell apoptosis (Fig. 2C). In addition, the levels of apoptosis-related proteins, including cleaved caspase-3, cleaved caspase-9, Bax and Bcl-2, were evaluated by western blotting. The result indicated that overexpression of miR-222 significantly promoted the expression of cleaved caspase-3, cleaved caspase- 9 and Bax and decreased the level of Bcl-2 
A

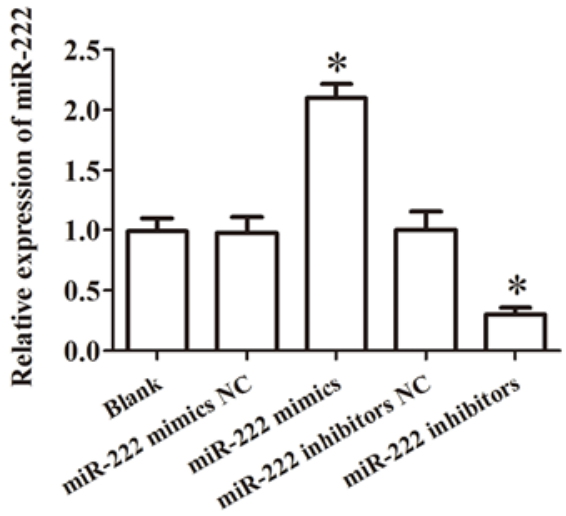

B

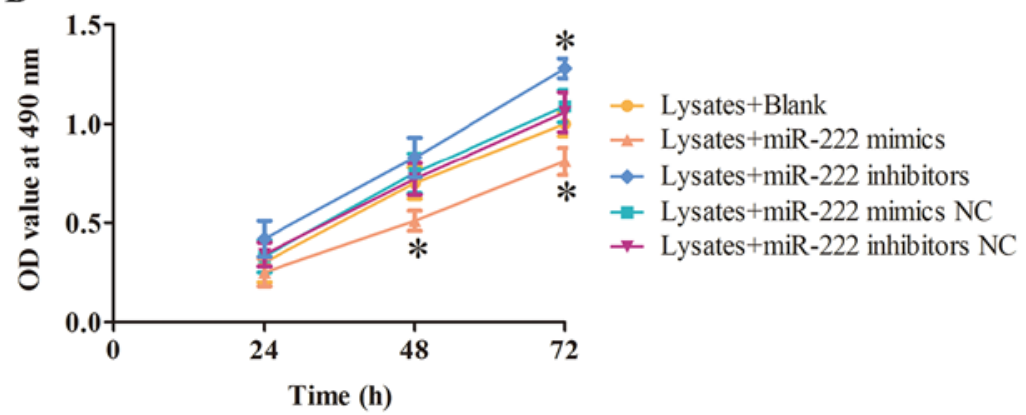

C
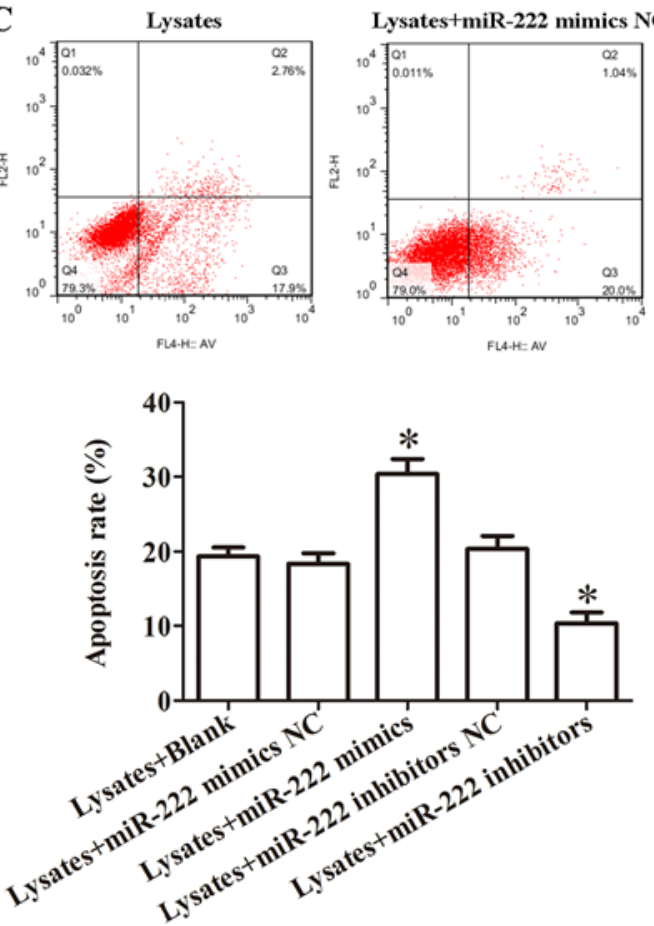
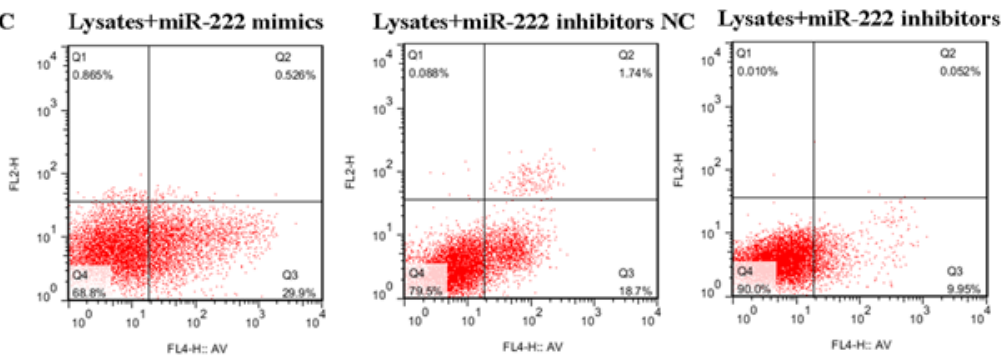

D

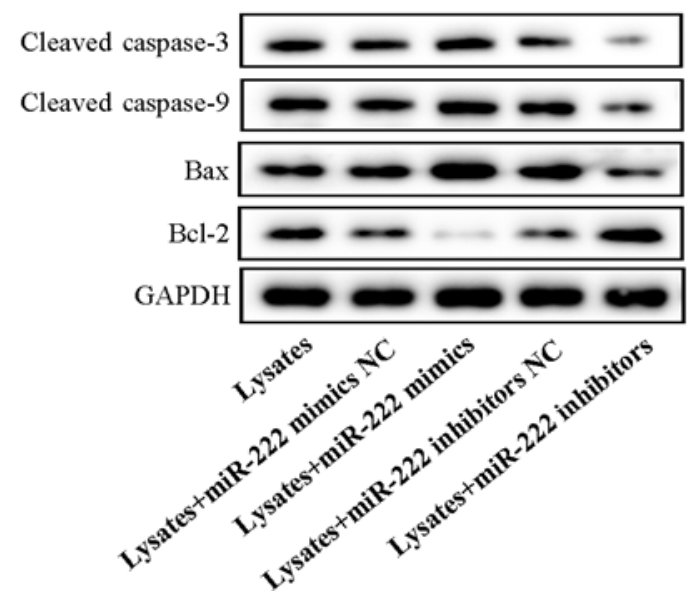

Figure 2. miR-222 regulates erythrocyte lysate-induced microglia cell viability and apoptosis. (A) Reverse transcription-quantitative PCR assay was performed to assess the transfection efficiency. (B) Cell viability was detected in erythrocyte lysate-induced microglia by a Cell Counting Kit-8 assay. (C) Effect of miR-222 on cell apoptosis was evaluated by flow cytometric analysis. (D) Levels of apoptosis-related proteins including cleaved caspase-3, cleaved caspase-9, Bax and Bcl-2 were evaluated by western blotting. " $\mathrm{P}<0.05$ vs. the corresponding NC group. NC, negative control; miR-222, microRNA-222.

in erythrocyte lysate-induced microglia cells, however, miR-222 inhibitors produced the opposite effect (Fig. 2D).

miR-222 regulates inflammatory response in erythrocyte lysate-induced microglia cells. The expression of cytokines in microglia exposed to erythrocyte lysates was assessed by ELISA. As revealed in Fig. 3, miR-222 mimics significantly promoted the levels of IL- 6 , TNF- $\alpha$, MCP-1 and IL- $1 \beta$ compared to the $\mathrm{NC}$ group in microglia exposed to erythrocyte lysates, while inhibition of miR-222 significantly suppressed the expression of these cytokines. These results demonstrated that miR-222 could regulate inflammatory response in erythrocyte lysate-induced microglia cells.

miR-222 mediates brain damage in ICH mice. After being subjected to ICH for $72 \mathrm{~h}$, miR-222 expression, water content in the brains of mice, the expression of inflammatory cytokines and neurological injury were evaluated. The results demonstrated that the expression of miR-222 subjected to ICH significantly increased (Fig. 4A). In addition, the water content in the brains of mice and neurological injury in the ICH group were significantly increased when compared to the sham group, while inhibition of miR-222 clearly decreased the water content and neurological injury (Fig. 4B and C). To determine the contribution of miR-222 to inflammation, ELISA was performed to assess the levels of inflammatory factors in brain tissues, including IL- 6 , TNF- $\alpha$, MCP-1 and IL-1 $\beta$. The results demonstrated that the expression of IL-6, TNF- $\alpha$, MCP-1 and IL-1 $\beta$ were significantly increased in the ICH group compared to the sham group, while miR-222 inhibitors significantly decreased the expression of these cytokines (Fig. 4D). These results revealed that inhibition of miR-222 could ameliorate the neurological symptoms and improve brain function after $\mathrm{ICH}$. 

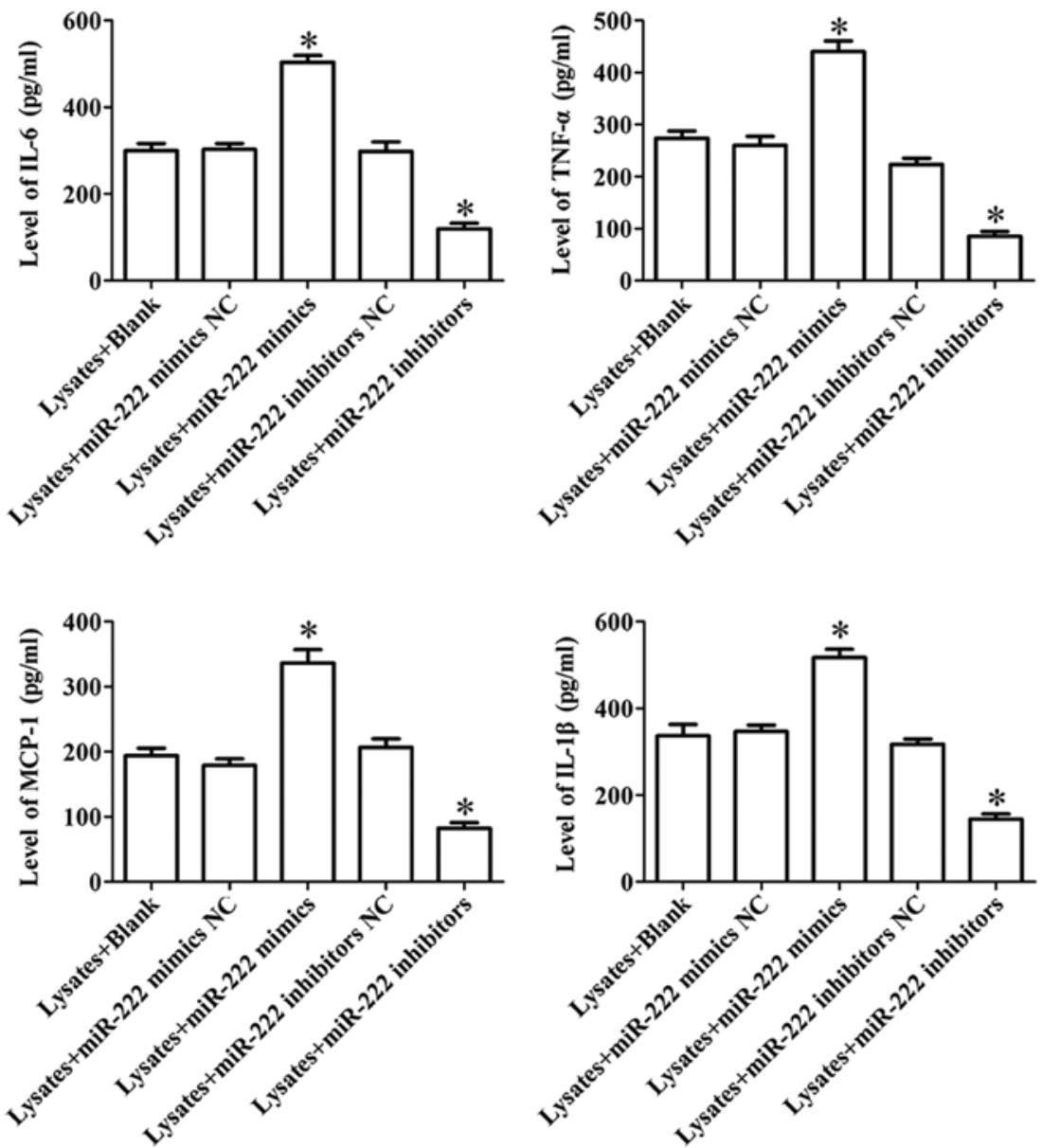

Figure 3. miR-222 regulates the inflammatory response in erythrocyte lysate-induced microglia cells. The expression of cytokines of microglia exposed to erythrocyte lysates was assessed by ELISA. " $\mathrm{P}<0.05$ vs. the corresponding $\mathrm{NC}$ group. NC, negative control.
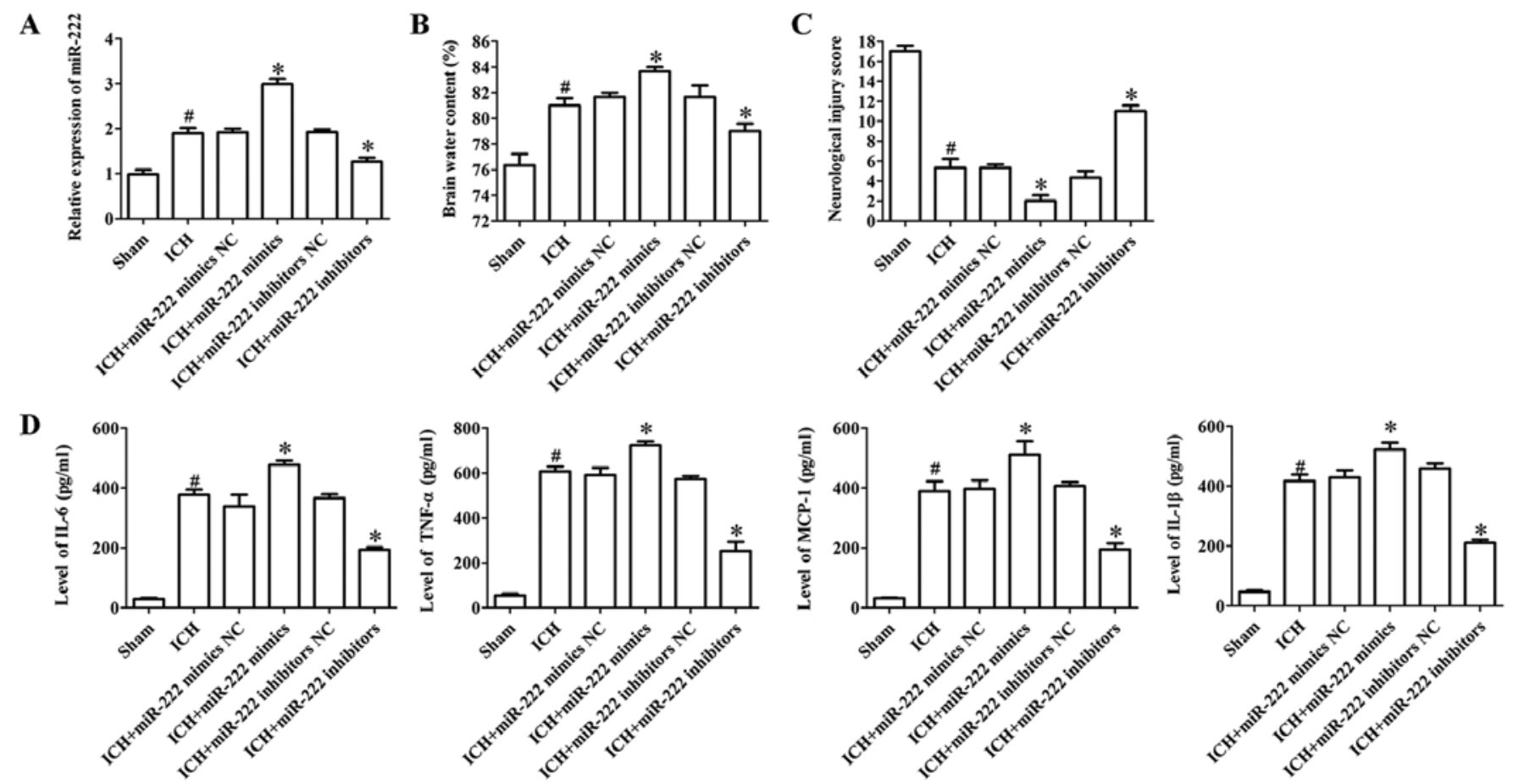

Figure 4. miR-222 mediates brain damage in ICH mice. (A) Reverse transcription-quantitative PCR assay was used to assess the relative expression of miR-222 in mice brain tissues. (B) Qater content and (C) neurological injury in the brains of mice were evaluated. (D) ELISA was performed to assess the levels of inflammatory factors in brain tissues, including IL-6, TNF- $\alpha, \mathrm{MCP}-1$ and IL-1 $\beta .{ }^{*} \mathrm{P}<0.05$ vs. the sham group; ${ }^{*} \mathrm{P}<0.05$ vs. the corresponding NC group. NC, negative control; ICH, intracerebral hemorrhage; miR-222, microRNA-222. 

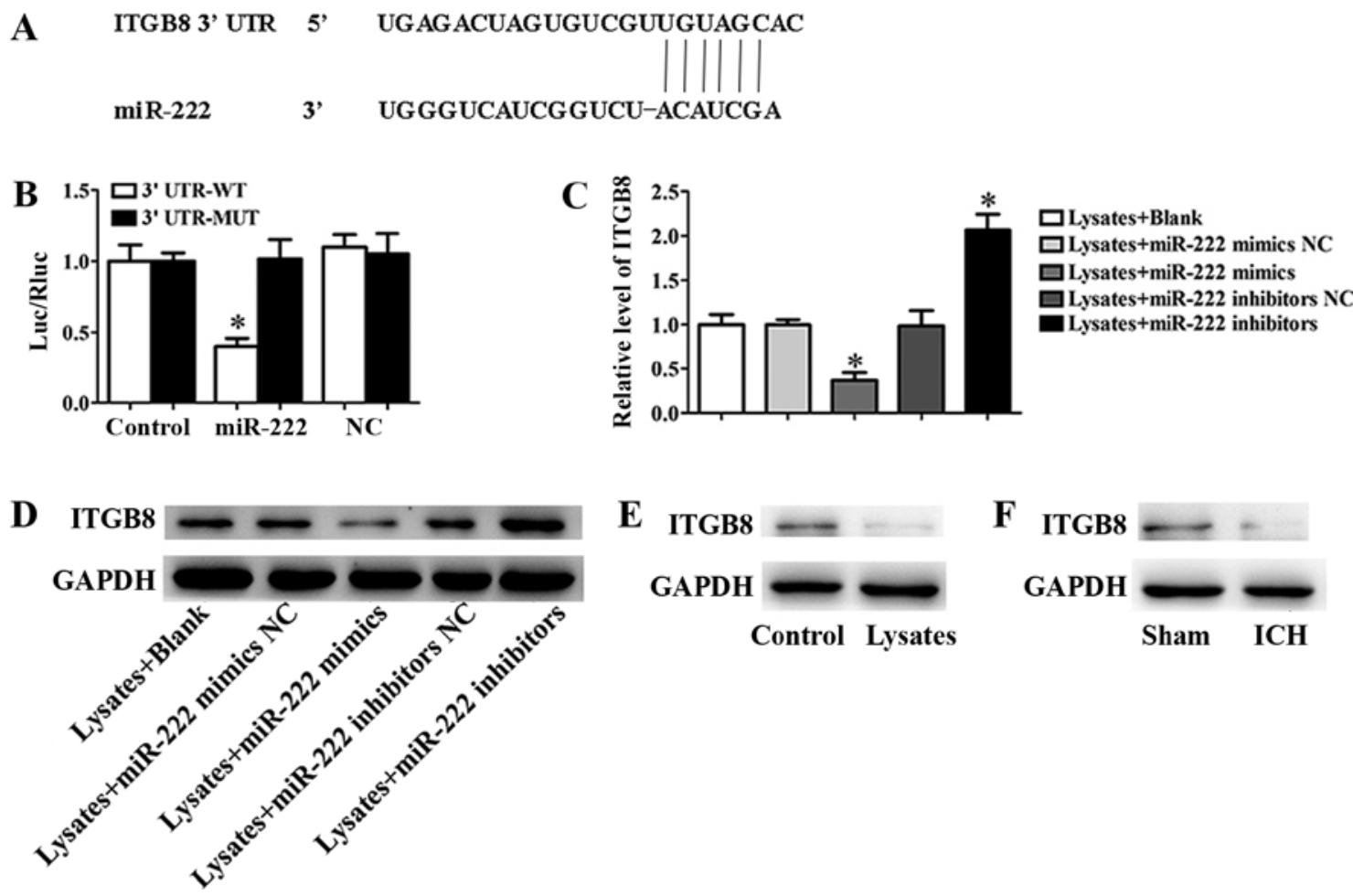

Figure 5. ITGB8 is a direct target of miR-222. (A) Target prediction program TargetScan (www.targetscan. org) was used to predict the target of miR-222. (B) To demonstrate that ITGB8 was a direct target of miR-222, a dual-luciferase reporter system assay was performed. (C) Reverse transcription-quantitative PCR and (D) western blotting assays were performed to evaluate the expression of miR-222. (E) A western blotting assay was performed to assess the expression of ITGB8 in microglia cells. (F) A western blotting assay was performed to assess the expression of ITGB8 in mice. "P<0.05 vs. the corresponding NC group. ITGB8, integrin subunit $\beta 8$; NC, negative control; ICH, intracerebral hemorrhage; miR-222, microRNA-222; UTR, untranslated region; WT, wild-type; MUT, mutant.

ITGB8 is a direct target of $m i R-222$. According to the target prediction program TargetScan, the 3'-UTR of ITGB8 mRNA contains a putative miR-222 target sequence (Fig. 5A). To demonstrate that ITGB8 was a direct target of miR-222 in microglia, a dual-luciferase reporter system was performed. The result revealed that co-expression with miR-222 mimics significantly inhibited the activity of a firefly luciferase reporter containing wild-type ITGB8 3'-UTR, while this was not detected on a reporter with a mutated ITGB8 3'-UTR (Fig. 5B). In addition, the results of qRT-PCR and western blot assay revealed that miR-222 mimics significantly inhibited the expression of ITGB8, however, inhibition of miR-222 could significantly promote this expression (Fig. 5C and D). In addition, erythrocyte lysates significantly inhibited the expression of ITGB8 in microglia cells (Fig. 5E). Moreover, in the ICH mice, the expression of ITGB8 was markedly downregulated compared to the sham group (Fig. 5F).

Inhibition of miR-222 promotes erythrocyte lysate-induced microglia cell viability, reduces apoptosis and inflammatory response by targeting ITGB8. To identify the role of ITGB8 in the miR-222-mediated inflammatory response, ITGB8 expression was promoted by pcDNA3.1. The result of qRT-PCR indicated that pcDNA3.1-ITGB8 significantly promoted the expression of ITGB8, while pcDNA3.1-NC did not increase this expression (Fig. 6A). Furthermore, a cell viability assay revealed that pcDNA3.1-ITGB8 clearly promoted erythrocyte lysate-induced microglia cell viability, and pcDNA3.1-ITGB8 relieved the decrease of cell viability of the miR-222 mimics
(Fig. 6B). In addition, overexpression of ITGB8 significantly promoted a reduction of erythrocyte lysate-induced cell apoptosis and co-transfection with miR-222 mimics significantly inhibited the decrease of cell apoptosis cause by overexpression of ITGB8 (Fig. 6C). Furthermore, pcDNA3.1-ITGB8 significantly inhibited the levels of inflammatory factors including IL-6, TNF- $\alpha$, MCP-1 and IL-1 $\beta$, and pcDNA3.1-ITGB8 could suppress the increase in the levels of inflammatory factors caused by miR-222 mimics (Fig. 7). These results indicated that inhibition of miR-222 promoted erythrocyte lysate-induced microglia cell viability, reduced apoptosis and inflammatory response by targeting ITGB8.

\section{Discussion}

$\mathrm{ICH}$, a common type of stroke with high mortality and morbidity, is one of the leading causes of death worldwide (23). Previous studies have revealed the involvement of miR-222 in the regulation of angiogenesis and inflammation $(24,25)$. Therefore, it was hypothesized that miR-222 may play a key role in the occurrence and development of ICH. In the present study, erythrocyte lysates could significantly promote the expression of miR-222 in microglia. Inhibition of miRNA-222 promoted erythrocyte lysate-induced microglia cell viability and decreased cell apoptosis. In addition, in ICH mice, miR-222 inhibitors could significantly decrease water content and neurological injury, as well as reduce the expression of inflammatory factors. Furthermore, ITGB8 was identified as a direct target of miR-222, and miR-222 could downregulate 

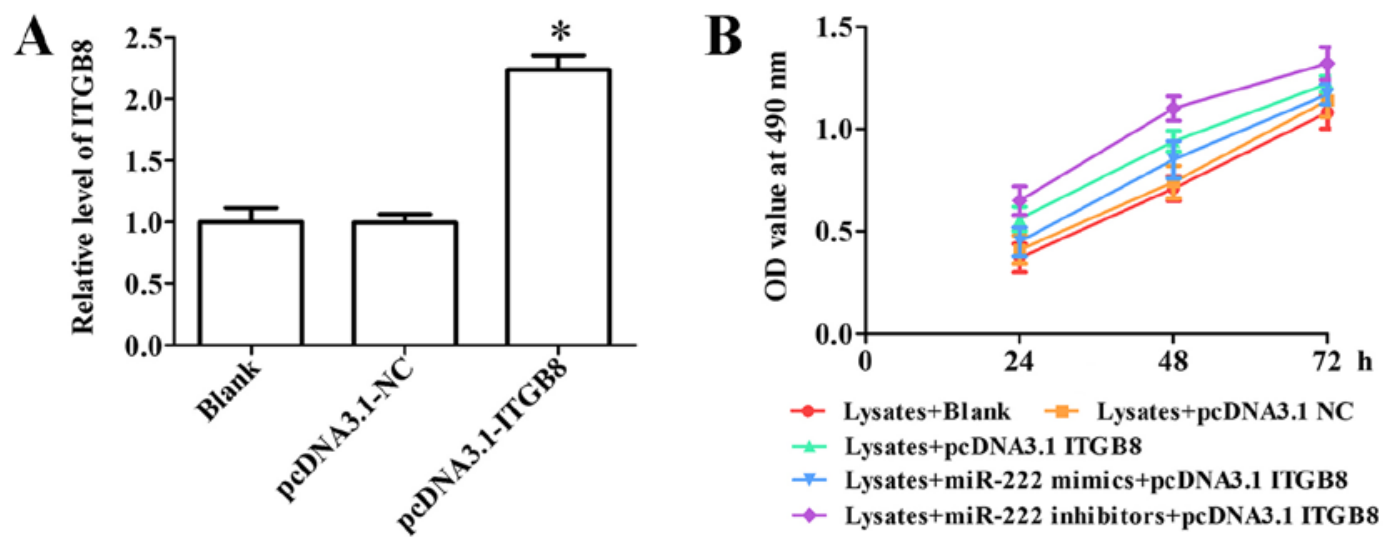

C

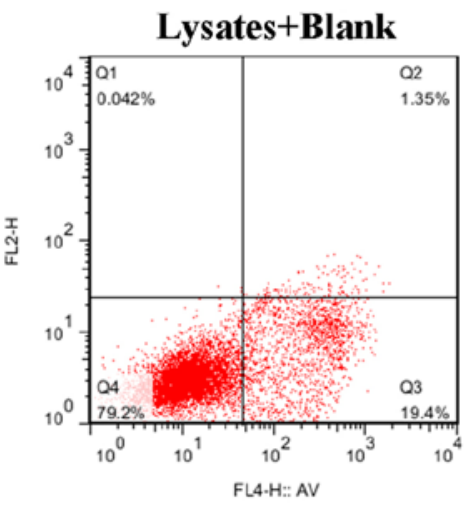

Lysates+pcDNA3.1 NC

Lysates+pcDNA3.1 ITGB8

Lysates+pcDNA3.1 ITGB8 +miR-222 mimics


\section{Lysates+pcDNA3.1 ITGB8} +miR-222 inhibitors
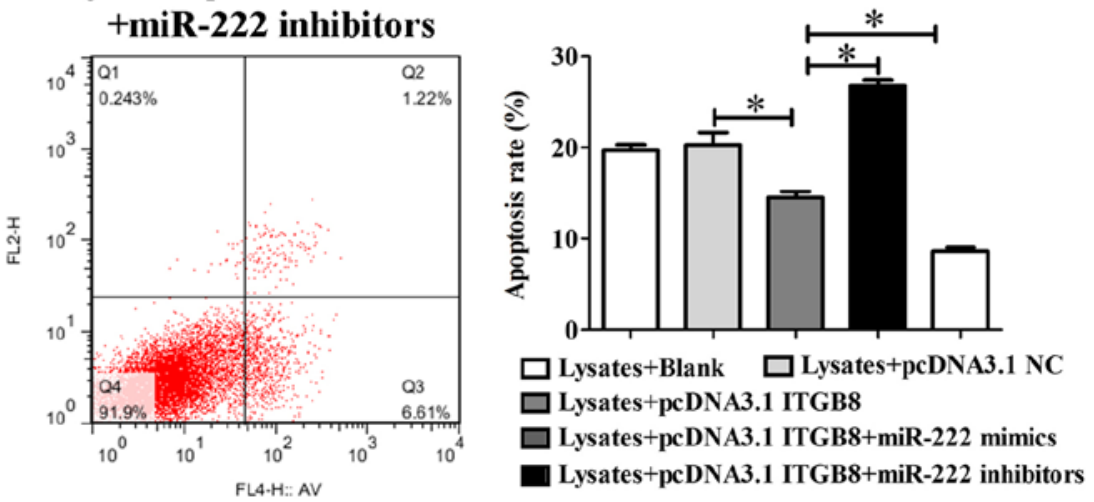

Figure 6. Inhibition of miR-222 promotes erythrocyte lysate-induced microglia cell viability and reduces apoptosis by targeting ITGB8. (A) A reverse transcription-quantitative PCR assay was performed to assess the transfection efficiency. ${ }^{*} \mathrm{P}<0.05$ vs. pcDNA3.1NC. (B) Cell viability was detected in erythrocyte lysate-induced microglia by a Cell Counting Kit-8 assay. (C) Effect of miR-222 and ITGB8 on cell apoptosis was evaluated by flow cytometric analysis. ${ }^{*} \mathrm{P}<0.05$. ITGB8, integrin subunit $\beta 8$; NC, negative control; miR-222, microRNA-222; OD, optical density.
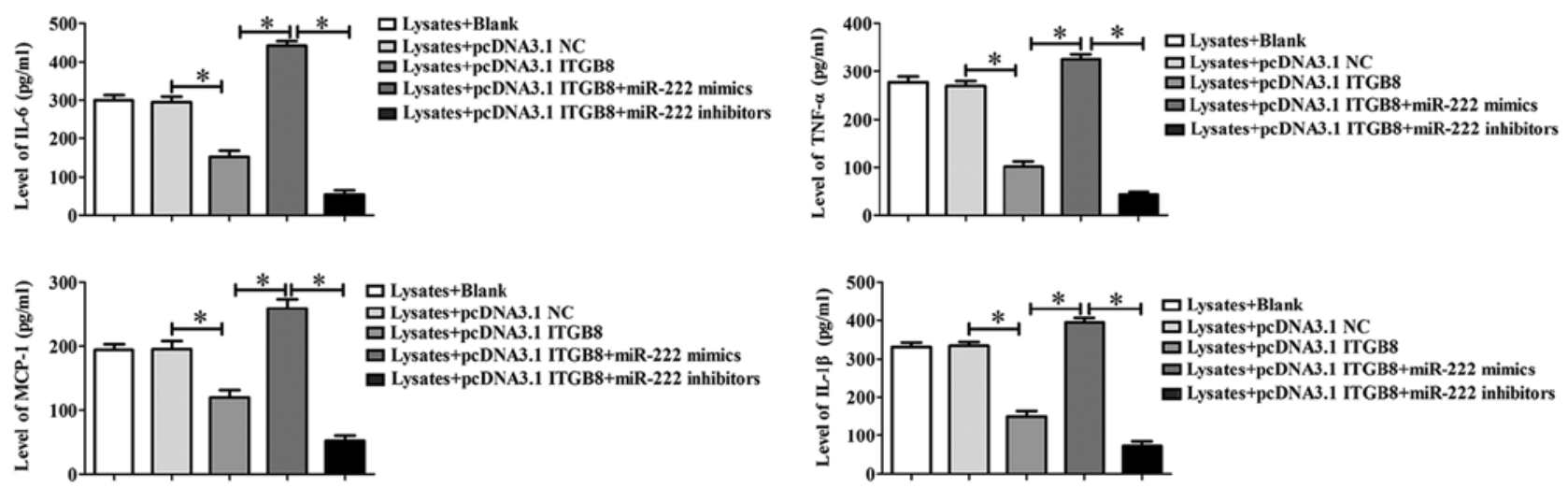

Figure 7. Inhibition of miR-222 inhibits inflammatory response by targeting ITGB8. The cytokine expression of microglia exposed to erythrocyte lysates was assessed by ELISA. ${ }^{*} \mathrm{P}<0.05$. ITGB8, integrin subunit $\beta 8$; NC, negative control; miR-222, microRNA-222. 
ITGB8 to inhibit inflammation, reduce brain edema and improve neurological functions.

After ICH, microglia are greatly active, and their reaction stimulated by hemorrhagic brain damage is extremely complex, which is far beyond the nutrition of neurons and the scope of denaturation and necrosis (26). Significant necrosis and apoptosis are often accompanied by neuronal cells around the hemorrhagic foci, and these necrotic lesions gather around a large number of activated microglia and can cause the release of a series of cytokines including NO, TNF and IL-6 (27). Therefore, the role of its mediated inflammatory response in the progression of $\mathrm{ICH}$ has been gradually recognized. Qureshi et al (28) revealed that there were 10 cases of apoptosis in tissue specimens in the perihematoma region in 12 patients with ICH. Apoptosis was observed in the specimens obtained at days 1,2 and 5 after the onset of symptoms. The average percentage of apoptotic cells around the hematoma was $38 \%$, while the average percentage of necrotic cells was only $25 \%$, indicating that apoptosis was the main form of cell death around the hematoma. In in vitro experiments, it was revealed that the production of NO in activated microglia significantly increased and the inducible NO synthase notably increased in the cells. Concurrently, NO mediated mitochondrial DNA damage and led to apoptosis (29). In consistence with these findings, our results revealed that erythrocyte lysates could significantly suppress cell viability and induce cell apoptosis in microglia, and promote the expression of cytokines. In addition, erythrocyte lysates could significantly promote the expression of miR-222 in microglia. Furthermore, it was revealed that inhibition of miRNA-222 could reverse erythrocyte lysate-induced damage in microglia.

The formation of cerebral edema after intracerebral hemorrhage is the most important pathological change of intracerebral hemorrhage, which is also the key factor leading to the deterioration of clinical cerebral hemorrhage (30). Clinical studies have revealed that the peak of death from cerebral hemorrhage occurs in the first few days after the onset of symptoms, which may be related to progressive brain edema formation (31). Cerebral edema develops rapidly after intracerebral hemorrhage, occurs within 1-2 $\mathrm{h}$ after hemorrhage, progressively aggravates, peaks at $24 \mathrm{~h}$, and begins to be absorbed for 4-5 days (32). The study of the mechanism of secondary cerebral edema after intracerebral hemorrhage can help the clinical treatment of cerebral hemorrhage patients, reduce secondary injury around the hematoma, reduce the mortality and disability rate of cerebral hemorrhage, and improve the quality of life of the patients (33). In the present study, the results revealed that miR-222 inhibitors could significantly decrease the water content and neurological injury, as well as reduce the expression of inflammatory factors in $\mathrm{ICH}$ mice.

ITGB8 is an important member of the integrin family, members of which are mediators of the interactions between cells and the matrix (34). Previous studies have revealed that ITGB8 in perivascular astrocytes plays an important role in regulating brain vessel homeostasis through modulation of TGF- $\beta$ activation and expression of TGF- $\beta$-responsive genes that promote vessel differentiation and stabilization (35). In addition, Ma et al (36) revealed that ITGB8 deficiency enhanced the formation of dysplastic vessels and hemorrhage. Herein, ITGB8 was identified as a direct target of miR-222, and miR-222 could downregulate ITGB8 to inhibit inflammation, increase cell viability and decrease cell apoptosis in erythrocyte lysate-induced microglia.

In conclusion, it was revealed in the present study, that miR-222 suppressed ITGB8 production by directly binding to its 3'-UTR, which reduced neuronal inflammation and improved neuronal function.

\section{Acknowledgements}

Not applicable.

\section{Funding}

No funding was received.

\section{Availability of data and materials}

All data generated or analyzed during this study are included in this published article.

\section{Authors' contributions}

YYB and JZN performed the experiments and analyzed the data. JZN was a major contributor in writing the manuscript. YYB and JZN both read and approved the final manuscript and agree to be accountable for all aspects of the research in ensuring that the accuracy or integrity of any part of the work are appropriately investigated and resolved.

\section{Ethics approval and consent to participate}

Experiments were performed according to animal care guidelines approved by The Animal Ethics Committee of Nanjing Medical University, and animals were treated in accordance with The Guidelines of the United States National Institutes of Health.

\section{Patient consent for publication}

Not applicable.

\section{Competing interests}

The authors declare that they have no competing interests.

\section{References}

1. Wang MD, Wang Y, Xia YP, Dai JW, Gao L, Wang SQ, Wang HJ, Mao L, Li M, Yu SM, et al: High serum MiR-130a levels are associated with severe perihematomal edema and predict adverse outcome in acute ICH. Mol Neurobiol 53: 1310-1321, 2016.

2. Min H, Jang YH, Cho IH, Yu SW and Lee SJ: Alternatively activated braininfiltrating macrophages facilitate recovery from collagenase-induced intracerebral hemorrhage. Mol Brain 9: 42, 2016.

3. Rodríguez JA, Sobrino T, López-Arias E, Ugarte A, Sánchez-Arias JA, Vieites-Prado A, de Miguel I, Oyarzabal J, Páramo JA, Campos F, et al: CM352 reduces brain damage and improves functional recovery in a rat model of intracerebral hemorrhage. J Am Heart Assoc 6: pii: e006042, 2017.

4. Keep RF, Hua $\mathrm{Y}$ and Xi G: Intracerebral haemorrhage: Mechanisms of injury and therapeutic targets. Lancet Neurol 11: 720-731, 2012. 
5. Sheth $\mathrm{KN}$ and Rosand J: Targeting the immune system in intracerebral hemorrhage. JAMA Neurol 71: 1083-1084, 2014.

6. Wu H, Zhang Z, Li Y, Zhao R, Li H, Song Y, Qi J and Wang J: Time course of upregulation of inflammatory mediators in the hemorrhagic brain in rats: Correlation with brain edema. Neurochem Int 57: 248-253, 2010.

7. Zhang Z, Zhang Z, Lu H, Yang Q, Wu H and Wang J: Microglial polarization and inflammatory mediators after intracerebral hemorrhage. Mol Neurobiol 54: 1874-1886, 2017.

8. Zhao X, Wu T, Chang CF, Wu H, Han X, Li Q, Gao Y, Li Q, Hou Z, Maruyama T, Zhang J and Wang J: Toxic role of prostaglandin E2 receptor EP1 after intracerebral hemorrhage in mice. Brain Behav Immun 46: 293-310, 2015

9. Ge Y, Yan X, Jin Y, Yang X, Yu X, Zhou L, Han S, Yuan Q and Yang M: fMiRNA-192 and miRNA-204 directly suppress lncRNA HOTTIP and interrupt GLS1-Mediated glutaminolysis in hepatocellular carcinoma. PLoS Genet 11: e1005726, 2015.

10. Martignani E, Miretti S, Accornero P and Baratta M: miRNAs highlights in stemand cancer cells. Mini Rev Med Chem 11: 1165-1182, 2011.

11. Veremeyko T, Siddiqui S, Sotnikov I, Yung A and Ponomarev ED: IL-4/IL-13-dependent and independent expression of miR-124 and itscontribution to M2 phenotype of monocytic cells in normal conditions andduring allergic inflammation. PLoS One 8: e81774, 2013.

12. Moore CS, Rao VT, Durafourt BA, Bedell BJ, Ludwin SK, Bar-Or A and Antel JP: miR-155 as a multiple sclerosis-relevant regulator of myeloid cellpolarization. Ann Neurol 74: 709-720, 2013.

13. Xi T, Jin F, Zhu Y Wang J, Tang L, Wang Y, Liebeskind DS and He Z: MicroRNA-126-3p attenuates blood-brain barrier disruption, cerebral edema and neuronal injury following intracerebral hemorrhage by regulating PIK3R2 and Akt. Biochem Biophys Res Commun 494: 144-151, 2017.

14. Yu A, Zhang T, Duan H, Pan Y, Zhang X, Yang G, Wang J, Deng Y and Yang Z: MiR-124 contributes to M2 polarization of microglia and confers brain inflammatory protection via the $\mathrm{C} / \mathrm{EBP}-\alpha$ pathway in intracerebral hemorrhage. Immunol Lett 182: 1-11, 2017.

15. Galardi S, Mercatelli N, Giorda E, Massalini S, Frajese GV, Ciafre SA and Farace MG: miR-221 and miR-222 expression affects the proliferation potential of human prostate carcinoma cell lines by targeting p27Kip1. J Biol Chem 282: 23716-23724, 2007.

16. Sun T, Wang X, He HH, Sweeney CJ, Liu SX, Brown M, Balk S, Lee GS and Kantoff PW: miR-221 and miR-222 expression affects the proliferation potential of human prostate carcinoma cell lines by targeting p27Kip1. Oncogene 33: 2790-2800, 2014.

17. Zhou Z, Zhou L, Jiang F, Zeng B, Wei C, Zhao W and Yu D: Downregulation of miR-222 induces apoptosis and cellular migration in adenoid cystic carcinoma cells. Oncol Res 25: 207-214, 2017

18. Kinoshita K, Matsumoto K, Kurauchi Y, Hisatsune A, Seki T and Katsuki H: A Nurrl agonist amodiaquine attenuates inflammatory events and neurological deficits in a mouse model of intracerebral hemorrhage. J Neuroimmunol 330: 48-54, 2019.

19. Chen D, Wu X, Zhao J and Zhao X: MicroRNA-634 functions as a tumor suppressor in pancreatic cancer via directly targeting heat shock-related 70-kDa protein 2. Exp Ther Med 17: 3949-3956, 2019.

20. Livak KJ and Schmittgen TD: Analysis of relative gene expression data using real-time quantitative PCR and the 2(-Delta Delta C(T)) method. Methods 25: 402-408, 2001.

21. Yu A, Duan H, Zhang T, Pan Y, Kou Z, Zhang X, Lu Y, Wang S and Yang Z: IL-17A promotes microglial activation and neuroinflammation in mousemodels of intracerebral haemorrhage. Mol Immunol 73: 151-157, 2016.
22. Wasserman JK, Zhu X and Schlichter LC: Evolution of the inflammatoryresponse in the brain following intracerebral hemorrhage and effects of delayedminocycline treatment. Brain Res 1180: 140-154, 2007.

23. Xie WJ, Yu HQ, Zhang Y, Liu Q and Meng HM: CD163 promotes hematoma absorption and improves neurological functions in patients with intracerebral hemorrhage. Neural Regen Res 11: $1122-1127,2016$

24. Corsten MF, Heggermont W, Papageorgiou AP, Deckx S, Tijsma A, Verhesen W, van Leeuwen R, Carai P, Thibaut HJ, Custers K, et al: The microRNA-221/-222 cluster balances the antiviral and inflammatory response in viral myocarditis. Eur Heart J 36: 2909-2919, 2015.

25. Ribeiro-Rodrigues TM, Laundos TL, Pereira-Carvalho R, Batista-Almeida D, Pereira R, Coelho-Santos V, Silva AP, Fernandes R, Zuzarte M, Enguita FJ, et al: Exosomes secreted by cardiomyocytes subjected to ischaemia promote cardiac angiogenesis. Cardiovasc Res 1113: 1338-1350, 2017.

26. Zhao X, Sun G, Ting SM, Song S, Zhang J, Edwards NJ and Aronowski J: Cleaning up after ICH: The role of Nrf2 in modulating microglia function and hematoma clearance. J Neurochem 133: 144-152, 2015.

27. Yang Z, Zhao T, Zou Y, Zhang JH and Feng H: Curcumin inhibits microglia inflammation and confers neuroprotection in intracerebral hemorrhage. Immunol Lett 160: 89-95, 2014.

28. Qureshi AI, Suri MF, Ostrow PT, Kim SH, Ali Z, Shatla AA, Guterman LR and Hopkins LN: Apoptosis as a form of cell death in intracerebral hemorrhage. Neurosurgery 52: 1041-1047, 2003.

29. Beufler B: Innate immunity: An overview. Mol Immunol 40: 845-859, 2004

30. Gebel JM, Brott TG, Sila CA, Tomsick TA, Jauch E, Salisbury S, Khoury J, Miller R, Pancioli A, Duldner JE, et al: Decreased perihematomal edema in thrombolysis-related intracerebral hemorrhage compared with spontaneous intrscerebral hemorrhage. Stroke 31: 596-600, 2000

31. Xi G, Wagner KR, Keep RF, Hua Y, de Courten-Myers GM, Broderick JP, Brott TG and Hoff JT: Role of blood clot formation on early edema development after experimental intracerbral hemorrhage. Stroke 29: 2580-2586, 1998.

32. Wei P, You C, Jin H, Chen H and Lin B: Correlation between serum IL-1beta levels and cerebral edema extent in a hypertensive intracerebral hemorrhage rat model. Neurol Res 36: 170-175, 2014.

33. $\mathrm{Hu} \mathrm{YL}$, Wang $\mathrm{H}$, Huang $\mathrm{Q}$, Wang $\mathrm{G}$ and Zhang $\mathrm{HB}$ : MicroRNA-23a-3p promotes the perihematomal edema formation after intracerebral hemorrhagevia ZO-1. Eur Rev Med Pharmacol Sci 22: 2809-2816, 2018.

34. Kumar V, Soni UK, Maurya VK, Singh K and Jha RK: Integrin beta8 (ITGB8) activates VAV-RAC1 signaling via FAK in the acquisition of endometrial epithelial cell receptivity for blastocyst implantation. Sci Rep 7: 1885, 2017.

35. Cambier S, Gline S, Mu D, Collins R, Araya J, Dolganov G, Einheber S, Boudreau N and Nishimura SL: Integrin alpha(v) beta8-mediated activation of transforming growth factor-beta by perivascular astrocytes: An angiogenic control switch. Am J Pathol 166: 1883-1894, 2005.

36. Ma L, Shen F, Jun K, Bao C, Kuo R, Young WL, Nishimura SL and Su H: Integrin $\beta 8$ deletion enhances vascular dysplasia and hemorrhage in thebrain of adult alk1 heterozygous mice. Transl Stroke Res 7: 488-496, 2016.

This work is licensed under a Creative Commons Attribution-NonCommercial-NoDerivatives 4.0 International (CC BY-NC-ND 4.0) License. 